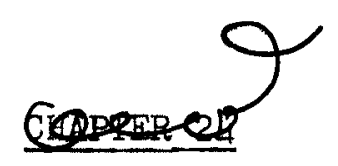

\title{
SIMULATION OF SHOCK AND VIBRATION ENVIRONMENTS
}

In simulating shock and vibration environments, the degree of realism in simulation which should be sought, and the degree which has been achieved are both questions worthy of serious consideration. There is a fairly common tendency to strive for nearly one hundred percent realism, regardless of the cost of or the need for this result. On the otner nard, there is all too common a tendency to accept a particular test as if it wcre one hundred percent realistic, without measuring to see $i^{r}$ this is true, or without determining whether the damare processes involved in the test are the same ones thet occur in the service environment. If in this chapter we can develop a clear picture of what constitutes realism in environmental simulation, then the foundation can be laid for the reader to judge how much realism he needs in a particular case, and how much he can afford to spend to achieve such realism. Realism of environmental testing requires that the damage processes in the test be identical with the damace processes in the service environment, both in nature, rate, and extent. 24-1. Purpose of Simulation. Usually the simulation of shock and vibration environments is intended to accomplish one of the following results:

In a basic study of the mecharisms of response to shcck, it would be desirable to generate several diflerent kinds of shock pulse and apply them to various structures and components with proper measurements of both the applied shock and the component response as discussed ir Chartor

\section{$\cos 001$}




\section{DISCLAIMER}

This report was prepared as an account of work sponsored by an agency of the United States Government. Neither the United States Government nor any agency Thereof, nor any of their employees, makes any warranty, express or implied, or assumes any legal liability or responsibility for the accuracy, completeness, or usefulness of any information, apparatus, product, or process disclosed, or represents that its use would not infringe privately owned rights. Reference herein to any specific commercial product, process, or service by trade name, trademark, manufacturer, or otherwise does not necessarily constitute or imply its endorsement, recommendation, or favoring by the United States Government or any agency thereof. The views and opinions of authors expressed herein do not necessarily state or reflect those of the United States Government or any agency thereof. 


\section{DISCLAIMER}

Portions of this document may be illegible in electronic image products. Images are produced from the best available original document. 
In such a study, it would be necessary to determine the effect of both the time-dependent parameters and the amplitude-dependent parameters. For example, the ratios of the rise-time, the dwell-tine, and the decaytime of the shock pulse to the natural periods of the responding structure are important parameters (see Fig. 24.1). The rate of rise or decay in terms of impulse units per second, and the amplitude of the maximum pulse would also be important. During such a basic study, it would be further desirable to try to induce failures in the structures' or components, so that mechanisms of failure could be studied. It does not help to show that an item survived a given test if the margin to failure is unknown.

Similarly, in a basic study of the mechanisms of response to vibration, it would be desirable to generate-several different kinds of vibration and apply them to various structures and components with proper measurements of both the applied vibration and the component response. In this case, and for shock too, the characteristics of the tested component affect the capacity of the vibration generator to produce what It wants to produce, as discussed in Chapter . In a vibration study, it would be important to be able to generate pure sinusoids of a wide range of frequency and amplitude, to generate multiple sinusoids of two or three or more distinct frequencies, and to generate a continuous spectrum whose spectral density versus frequency curve could be controlled. Various kinds of vibration spectra and their effect upon structures are discussed in Chapter - For such vibration studies, it would again be important to try to induce failures, so that mechanisms of failure could be studied. 
These basic studies of shock and vibration would be aimed toward the measurement, understanding, and quantitative definition of what damage processes are involved, and how they develop and accumulate into an eventual fallure.

Another purpose for simulating shock and vibration environments is to determine means for improving a contemplated design or to prove the adequacy of an actual prototype design. This kind of testing is much more limited in scope and depth of inquiry than the basic studies previously discussed. For example, it may be desired to determine which of two chassis structures would produce the least vibration excitation for an attached component over a specific frequency range in which the component was particularly sensitive. To do this, it would only be necessary to measure the magnification factor versus frequency in the range of interest for the two chassis. In another case, it may be desired to determine whether a particular chassis and the components mounted upon it can survive a specific shock pulse. To do this would simply require the application of the specific shock to the chassis. However, in both of these cases, it would be wise to carry the tests to failure by increasing the vibration or shock amplitude so that some measure of the margin to failure could be obtained. Caution must be used, however, lest the failures induced be a result of damage accumulation rather than a proper measure of the margin to failure for a single shock.

Once production runs of an item have been started, but before fullscale production is under way, it is desirable to determine whether the production units can pass the required environmental tests. These tests 
then constitute the acceptance tests of the item, and they usually would be carried out to suit a purchase specification rather than to achieve some specific level of realism in simulation of the service environment. It is in this area, purchase specifications, that a serious inquiry into the extent of realism needed and achieved by the tests would be most profitable. There are all too many test specifications now accepted without question which are more justifiable on the basis of history and inertia against change than they are justifiable as proper simulations of service environments.

After full-scale production is under way, tests for the purpose of quality control are in order. These tests may make no attempt at a high level of simulation of a service environment, so long as they serve the purpose of maintaining quality of production. In one case, the quality control test applied to automobile radios consisted of two blows of a hard rubber mallet applied to the chassis in each of the three principal directions. It had been found that, if the radio still operated after this test, it would then be satisfactory for automobile service. There is no doubt that some of our presently popular tests are more in the nature of this hammer test for quality control than they are environmental tests. To summarize, the purpose of simulation of shock and vibration environments is to produce in the tested item a process of damage which is related in some useful and known way to the damage which is expected from the service environment. 
24-2. Principles Involved in Simulation. In order to develop a set of principles for simulation of shock and vibration environments, let us consider some of the things that are known about the effect of shock and vibration. For this purpose, a list of observations to which the reader can add from his own experience will help to define the problem.

a. In cases of severe shock on an item of equipment, it is common for the whole equipment to be torn loose from its fastenings or for sections of the equipment to tear loose from their fastenings. It is also common for permanent deformation of the structural frame to occur, so that alignments and clearances are lost. It is relatively rare for an outright fracture to occur in a structural part away from its ends. b. Electrical equipment under shock may have plate-to-grid shorts in vacuum tubes, opening relays, and broken condenser leads. c. Mechanical equipment under shock may have gear shafts bent out of line, gyro balance destroyed by shaft bending or gimbal damage, and gear teeth broken.

d. Hydraulic equipment under shock may develop leaks, usually at joints.

e. Under vibration, all of the above failures may occur, with some additional phenomena which are peculiar to vibration. In structural parts, fatigue failures appear, usually associated with a resonance. These failures are not always found at fastenings or ends of members, though there may be some tendency for the rate of incidence to be greater there. 
f. Electrical equipment under vibration may suffer from noise generation in vacuum tubes, relay chatter, and changes of electrical characteristics of various items.

g. Mechanical equipment under vibration may develop loose fastenings, gyros may precess, and servo systems may hunt or become unstable. From this partial list, it should be clear that a wide variety of damage processes are involved in shock and vibration. Furthermore, it should be clear that several different damage processes may be involved in the same piece of equipment, so that what may be a proper simulation of the damage process for one item may be quite wrong for a neighboring item. Hence for a new piece of equipment for which service experience is not available, it is often necessary to consider carefully the various items which would be sensitive to shock or vibration and then devise tests to determine their degree of sensitivity and their margin to failure. Such tests should have as their principal purposes the determination of the damage processes involved, the relationships between these damage processes and the applied environments, and the establishment of a test which produces the same damage process as the service environment. 24-3. Specific Damage Processes and Their Causes. Nearly all of the damage processes resulting from shock or vibration are associated with the relative movement of various parts of the equipment. Relative movements of parts of a structure produce strains which can lead to permanent deformation, progressive fracture or fatigue, or one-cycle fracture. To predict with any accuracy the amount of permanent deformation 
that a structure will sustain as a result of a shock or vibration is at best a difficult job, and is often impossible because of the non-linear stress-strain relationships that are involved. The effect of damping in such cases is usually unknown, also. Consequently, damage from excessive permanent deformation of a structure can best be determined by test. For items too large to test, an educated estimate is usually all that can be done.

The damage produced in structural materials by fatigue or progressive fracture has received more attention than almost any other fallure process for nearly a century. The state of knowledge in this fleld was discussed and summarized at a symposium in $1951^{1}$, and more recently in $1956^{2}$. 4 study of the papers in these two symposia will show that, while fatigue damage under steady sinusoidal load is relatively well understood and predictable, fatigue damage from non-sinusoidal or randomly varying loads is not yet a predictable thing. Since a service environment is more likely to consist of randomly varying loads than of steady simusoidal loads, a test simulation of the service environment may be the only way that the adequacy of a piece of equipment can be evaluated. Responses to randomly varying loads can be predicted, as discussed in Chapter, , but the damage resulting from these responses is not likely to be predictable for some time yet. There are several hypotheses 3,4 concerning the accumulation of fatigue damage, but these are not yet supported by adequate data. For items too large to test, then, we must again rely on an educated estimate of adequacy. 
For damage to structural materials by one-cycle fracture, only the simpler cases are presently predictable. For example, the energyabsorbing capacity of a bolt under relatively slow strain rates can be determined and used to predict the behavior of that bolt 5 under shock load. However, if the strain rates are high enough, the results may change appreciably6. Here again is an area in which damage processes are not well known or understood, so that a test simulation of the service environment may be required for effective evaluation of damage. Very often, the strains in a structure under shock or vibration induce strains in components mounted on the structure. Condensers and resistors which are mounted by their own leads have these leads strained by the deformation of the structure on which they mount. This strain can lead to fatigue failure of the leads, or it can produce changes in the electrical characteristics of the resistor or condenser. Both of these difficulties can be overcome by proper mounting of the item, so that the remedy for this damage process is found in proper design and mounting details. Simple tests will usually suffice in this case, and a high degree of simulation is not required.

In addition to the strain-produced effects of shock and vibration, there is another class of damage processes which result from the absolute motions of the various items and the effects of inertia on these motions. For example, a relay may be held in open or closed position either by spring force or by magnetic force. A motion response to shock or vibration then produces an acceleration of the relay andits contact arm. 
When the inertia force associated with the acceleration equals or exceeds the holding force, the relay arm will move and change its condition of open or closed. If the acceleration involved is periodically reversed or sinusoidal, then the relay will chatter. These are both instances of electrical damage from acceleration as such. If the holding force and inertia of the relay arm are known, then the failure is predictable if the acceleration excitation is predictable. A test simulation may be required to determine the acceleration excitation, however.

Another example of acceleration effects is the precession of a gyro caused by unidirectional acceleration or by periodically reversed acceleration. Usually a mechanism in the gyro itself can be found which causes this precession to be non-symmetric, so that the precession produces spurious guidance information as part of a damage process. It is likely that tests will be required to determine the gyro sensitivity, and these tests could well be culminated in a service environment simulátion.

Vacuum tubes under acceleration undergo relative motion of their elements so that the transconductance is changed. This produces either a change in operating characteristics or the generation of noise, or both, with resulting damage. With vacuum tubes, enough tests to generate statistical data are usually required, and again these could include a simulated service environment. 
Servo valves develop a bias or a dither under acceleration, so that the system does not follow the input signals properly and may even become unstable. This failure may be predictable in the same way that relay failure may be predictable, but test simulations may be needed to improve the prediction. 24-4. Formulation of Specific Simulation Requirements.

a. For a basic inquiry into vibration damage mechanisms. Since the foregoing discussion has pointed out that simulation in testing should be aimed at producing the same damage processes as the service environment does, it is appropriate to begin the discussion of this section with a consideration of the requirements for a basic inquiry into vibration damage mechanisms. For a start, we could assume that test specimens and their mounting equipment could be kept in the 10 to 20 pound weight range. This would be enough to allow for structural studies with small flexure specimens; groups of vacuum tubes, condensers, resistors, and small relays; free gyros and rate gyros; servo valves; and other elements that could be subject to a vibration environment.

To study damage processes in such items, it would be desirable to be able to provide uni-directional vibration excitation in several forms. First we would probably wish to test with sinusoidal excitation so that curves similar to Fig. 2 could be developed. If the curves of Fig. 2 showed more than one frequency of damage sensitivity, we would then wish to test with two or more sinusoids simultaneously so that curves similar to Fig. 3 could be developed. The next step would be to test with a flat spectrum of random vibration excitation (see Chapter 
so that curves similar to Fig. 4 could be developed. Is final steps, we would wish to evaluate the effects of varying the frequency and amplitude ratios of the simultaneous sinusoids involved in Fig.3, and then the effects of shaping the random vibration spectrum to match the curves of Fig. 2, its inverse, and other shapes.

The frequency ranges involved in such an inquiry need to extend from as low as 5 or $10 \mathrm{cps}$ to at least as much as 2500 or $3000 \mathrm{cps}$. Even higher frequencies may be needed for some items, such as subminiature vacuum tubes or transmitter crystals.

The acceleration amplitudes would need to be as great as 25 or $30 \mathrm{~g}$ for single and multiple sinusoids, and the root-mean-square integral of the random vibration spectrum might need to be as much as 25 or $30 \mathrm{~g}$ over the band from 10 to $3000 \mathrm{cps}$.

Now we can list the requirements for a testing machine to perform the foregoing set of tests as follows:

(1) Capable of testing weights in the 10 to 20-pound range.

(2) Capable of uni-directional vibration excitation in the frequency range from 5 or $10 \mathrm{cps}$ to 2500 or $300 \mathrm{cps}$.

(3) Capable of producing sinusoidal excitation, multiple sinusoids, and random vibration spectra, all in the 25 to $30 \mathrm{~g}$ range.

(4) Capable of varying amplitude and frequency ratios for multiple sinusoids, and of shaping random spectra.

(5) Servo control of excitation to maintain prescribed levels of input. 
When we compare these requirements with the capabilities of available equipment, we find that the frequency range requirement rules out all but the electro-dynamic vibration exciter. The control and spectrum requirements dictate the use of an amplifier for the power source, and a signal generator with a shaping network is the answer to the multiple-sinusoid and spectrum reauirements. These are both compatible with an electro-dynamic vibration exciter, and are commercially available. The same is true for the feedback servo control.

With regard to size of the exciter equipment, it is wise to realize ahead of time that: (I) however large the test equipment may be, sooner or later the occasion will arise when a contemplated test is too large for the equipment; (2) large capacity test equipment is used for more than half its capacity for a good bit less than half the time; (3) much useful work can be done by ingenious design of tests to fit existing equipment; (4) more good can be accomplished by making a start on the work with equipment which is on hand or can be readily scrounged than by waiting too long for the ultimate in test gear to arrive. With these precepts in mind, we can conclude that any electro-dynamic exciter from 10 to 10,000 pound force rating and powered by alternators or amplifiers. could be used to start such work. An excellent investigation could be carried to completion with either a 1000 to 1500 pound exciter powered by a $15 \mathrm{kva}$ amplifier, or with a 2500 to 3500 pound exciter powered by a $30 \mathrm{kva}$ amplifier. Both of these Items are commercially available, together with compatible signal generators, shaping networks, and servo-controls, so that designing a new machine should not be necessary for such work. 
b. For a basic inquiry into shock damage mechanisms.

The same discussion about weight range of specimens and kinds of specimens applies to a basic inquiry into shock damage mechanisms as to vibration damage in subsection a. It would be particularly helpful in a basic investigation if identical specimens could be used for shock and vibration tests, so that possible correlations of damage processes could be found.

To study shock damage processes in such specimens, it would be desirable to be able to produce uni-directional shock which could be controlled to give various shapes of shock pulses. A good place to start would be with half-sine-wave pulses of various time durations and amplitudes, to produce curves similar to Fig. 5. Ramp-shape puIses of acceleration could be used next to produce a similar set of data, and step velocity pulses likewise.

With regard to specific requirements, half-sine pulses would need to vary in duration from $10^{-4}$ seconds to perhaps 0.1 seconds, with amplitudes from about $1000 \mathrm{~g}$ for the short duration to $50 \mathrm{~g}$ or less for the long duration. Ramp-shape pulses would need rise-times comparable to the half-sine durations, and $g$ levels of similar magnitudes. Step velocity pulses might need to be as great as 500 in. per sec, but it might be possible to stop at half this value. 
For such a set of tests it would be possible to make a good start with a commercially available drop tester that could be adapted to produce half-sine pulses. Great care in instrumentation and detailed test technique would be needed, however, to insure that the shock pulses were truly measured in both form and magnitude. Enough work has been done in this field $7,8,9$ to illustrate the technical difficulties and to show that considerable development work may be necessary just to get ready for testing. Commercially available equipment is not likely to be directly applicable.

To produce ramp-shape pulses of acceleration, there are a few choices of approach through different forms of equipment that are presently available. Pneumatic-hydraulic testers have been developed ${ }^{10}$ which approximate a ramp-shape pulse and which might be adaptable for a basic investigation. Quick-acting rotary accelerators have also been developed 11,12 which give very nearly ramp-shape pulses, and these machines would probably be better for a beginning. Refinement or further development of these machines would Iikely be necessary as work progressed and problems became better understood.

For the production of step-velocity shock pulses, some form of the pendulum hammer type of tester 13,14 seems to offer the most promise. The Navy Hi-Impact Shock Machines have already received thorough measurement and study, so that much of the groundwork has been done and relatively less development work would be needed. 
As may be deduced from this discussion, the state of the art with regard to equipment for a basic inquiry into shock damage processes is not as good as for vibration. This may well be because of the greater difficulty of the problem, since comparable amounts of effort have gone into both fields. Consequently, the discussion of this subject has had to be more qualitative in regard to the specific choice of equipment for tests. It should be possible, however, to make a basic shock damage investigation parallel a vibration investigation, with similar presentation of data.

c. For a development comparison test.

Let us now consider a particular case in which it is desired to determine which of two designs of chassis and supporting structure will better perform the function of carrying the same electronic equipment in a guided missile. It is known that the missile and its equipment will have to withstand a launching shock which will be nearly ramp shape, with a rise-time in the 0.01 to 0.05 second range and a maximum acceleration of 10 to $30 \mathrm{~g}$. In addition it is suspected that the flight vibration which the supporting structure will see will be broadbanded or noise-type, with a root-mean-aquare value of as much as 10 or $20 \mathrm{~g}$. We will assume that proper equipment is available to produce, program, and monitor the input signal to the chassis, as well as to record the output signal from the chassis. This then allows us to establish as a criterion of comparison the deviation in output signal under vibration conditions versus static conditions. 
The next consideration would be that of the test fixture - how do we get hold of the chassis to test it? Ideally, the chassis should see the same mechanical impedance at its mounting to the test fixture as it sees at its mounting to the missile. This. is rarely possible, however, and the practical solution is to simulate enough structure around the chassis so that two or three structural elements intervene between the test fixture and the chassis. The test fixture is then designed to be either more or less stiff than the chassis-plus-structure assembly, in the same way that the rest of the missile would be more or less stiff. For our purpose of development comparison, greater refinement is hardly justifiable.

With this much of the problem lined up, the question now is how to test? Probably the most fruitful comparison can be made by testing each chassis in turn with a programmed sweep of sinusoidal vibration at constant $g$ level. This sweep should be slow enough to allow two things: time for resonances to build up to near maximum, and time for the input-output measurement program to provide significant measurements. The first requirement will be satisfied if the sweep rate is not faster than $f^{2} / Q^{2}$, where $f$ is the frequency in cps and $Q$ is the largest magnification factor that is to be given time to build up to its maximum response. This will insure that all resonances of magnification factor $Q$ or less will have time to rise to 95 percent of their maximum response. The second requirement will depend entirely on the nature of the input-output functions to be measured. For example, if the input is a radar signal for a guidance package, then the sweep must be slow enough to allow an evaluation of the guidance 
response once every bandwidth of a fixed number of cycles per second or of a variable bandwidth related to the frequency level, such as $\Delta f / f$. These two requirements may be incompatible, especially at the higher frequency ranges, and care is needed in working out the test program so that the higher frequency ranges are not unduly emphasized.

When the measurement and sweep rate programs have been adjusted to be as compatible as possible, testing can start. It is preferable to commence with low $g$ levels and progress in steps to higher levels until a definite failure is reached. A sequence of levels such as $1,2,5$, 10, etc., or $1,2,4,8$, etc. will require less testing and lead to satisfactory results in most cases. Whichever chassis gives less trouble during testing and withstands a higher $\mathrm{g}$ level before a definite failure is reached will usually be the more profitable one with which to continue development. The testing will undoubtedly disclose places where improvement in design is needed and may even point the way to the kind of change that will be most effective. Observation under stroboscopic light during testing is an invaluable aid in this respect. The testing machine needed for this phase of the work is an electrodynamic vibration exciter of large enough size to handle the chassis and test fixture. It can be alternator powered with an automatic sweep control, or amplifier powered. With an amplifierpowered machine, tests with random vibration could be made, but this is not essential for our purpose. 
It is quite satisfactory to base the design choice between two chassis on the above vibration test, and to complete development on the better one with vibration testing. This is so because whatever is done to improve broad-band vibration characteristics also improves shock response 15 . When the development by vibration test is complete, the shock test is likely to be routine and cause no further changes. A pneumatic-hydraulic shock tester or a quick-acting rotary accelerator of suitable size will do an adequate job of simulating a ramp-shape launching shock $10,11,12$ and complete the problem.

d. For an acceptance test.

Let us assume for this case that as true a simulation of enfironment as possible is desired, rather than the usual oversimplified specification test. For this purpose, we shall consider the acceptance testing of an autopilot free gyro for an airplane. It would be necessary to know the details of the mounting structure for the gyro in the airplane, and to have a series of acceleration measurements at that location with either a gyro in place or a mass and stiffness simulation of the gyro. This series of measurements should include normal and extraordinary operations of the airplane, smooth and rough takeoff and landing, and the like.

At this stage the question arises whether it is preferable to measure the mechanical impedance of the gyro mounting and attempt to design a test fixture which will simulate this impedance, or whether it is 
better to bilild as rigid a test fixture as is reasonable and count on being able to produce the proper excitation at the gyro mounting point. The former procedure would be feasible if the exciter characteristics were flat and would remain so even with the test fixture and gyro in place. It is doubtful whether this can be achieved with any existing equipment, even when the force rating of the exciter is 100 or 1000 times the weight of the fixture plus gyrol6. Since this is the case, the latter choice of building a rigid test fixture is the preferable one. This leads us then to the need to design a test fixture which will have no resonant frequency within a factor of ten or more of the lowest damagesensitive frequency of the gyro. If this can be done, then the selection of a vibration exciter boils down to: an electrodynamic exciter, amplifier powered, with compensating networks on the input to the amplifier capable of producing a flat (input to compensating networks equals output at gyro mounting) response at the gyro mounting when the gyro is replaced by a solid block of equal mass. This is quite specific and means that, for the usual gyro and test fixture weighing a total of about $30 \mathrm{lbs}$. , an electrodynamic exciter with a force rating in the 1000 to 1500 -pound range would be required, with an amplifier in the 15 to $20 \mathrm{kva}$ range, and compensating networks capable of producing about ten peaks or notches through the 10 to $2000 \mathrm{cps}$ range. (One problem to be guarded against is the effect of the magnetic field of the exciter upon the gyro. A degaussing attachment for the exciter can help solve this, as can a test fixture which puts the gyro in a weak part of the exciter's magnetic field.) 
Another important question must be answered before any testing can be started: what shall be the criterion for unacceptable gyro performance under vibration? It is wise to avoid the situation of performing an acceptance test without knowing specifically what kinds of things will be considered to be unacceptable (and acceptable) performance, and how much of these. For our problem, a good set of criteria would be that:

(1) The precession rate must be less than some specific number.

(2) The noise content of the output signal of the gyro must be less than some specific proportion of the total signal.

(3) The gyro must not tumble.

A final important question to be settled before testing can be started is: what test signal to use? To determine this some operations analysis is called for, so that the time and magnitude ratios to be assigned to takeoff, landing, smooth flight, rough maneuvers, and the like can be determined. From this analysis, a composite input signal for the test can be concocted which will reflect the design environment for the gyro.

Now we are finally ready to begin testing. It would be wise to test with intensity values of $\frac{1}{2}, 1,2,4$, etc. as compared to the design values, and to continue testing at each level until one of the above established damage conditions were reached. When the testing is completed, a comparison of time to damage with required design life will disclose whether or not the gyro is satisfactory.

If some stylized specification were to be used for the test requirements, then each of the foregoing steps could be modified to suit the specification. For example, measured flight environment would be replaced with specified 
values, the test fixture may be specified, the test environment may be spelled out in detail, and the acceptance criteria defined. This would effectively reduce the problem to a much simpler one which would require less engineering and judgment.

e. For a quality control test.

It is quite proper to reduce the amount of testing to be done on a single item or on groups of items as the production rate increases. This can be justified as statistical sampling techniques are used and shown to be effective. In addition the tests which prove to be non-definitive can be eliminated so that only those tests which in fact do determine adequacy of quality are retained. It is for such reasons (and for cost reduction) that quality control tests are usually simpler and less directly related to environment than the previously discussed kinds of tests.

As an example of a quality control test that was simple, was unrelated to environment, and did effectively control quality let us consider the subminiature vacuum tubes used in the VT fuzes. In the early stages of work with these tubes, it was found that the fuzes were failing because the glass envelopes of the tubes had failed, probably while the fuze was still in the gun barrel. A number of different kinds of tests were started in an attempt to cure the trouble. Tubes were vibration tested by mounting them on the voice coil of a dynamic loudspeaker (the forerunner of the present electrodynamic vibration exciter), and shock tested by dropping them down a chimney onto a concrete base. Examination of tubes that failed and tubes that did not fail did not disclose reasons for the difference until the glass envelopes were examined under polarized light. It then became clear that the failing tubes were the ones whose glass envelopes had 
residual stresses in them before the test. In time it was demonstrated that two very simple tests were enough to provide quality control: measurements of noise output of the tube while it was being vibrated, and polarized light examination of the glass envelope for residual stresses. When high-noise and high-stress tubes were eliminated, the service failure rate dropped to a satisfactorily low level and quality control was maintained. In this case no amount of environmental simulation could have served as effectively as the residual-stress check. So since quality control tests have as their sole purpose the elimination or minimization of service damage or fallure, such tests should be based upon studies of service damage and inquiry into the causes of this damage. If the cause of damage can be measured, or disclosed by testing, or caught by visual inspection, or reliably discovered by any other means, then these things constitute valid quality control tests. Careful simulation of environment is costly and usually can be justified only for research studies and for acceptance testing : development tests and quality control tests need not necessarily be good simulations of environment. 24-5. What to Do When It's Too Big to Test.

It is not unusual that simulation of shock or vibration environments would require impossibly large or expensive machines, or the available time is too short, or the design item is one of a kind and cannot be used for test purposes if there is any possibility of damage occurring. Such is the case for nuclear power plants: they are too big, too costly, one of a kind, and damage from testing could not be tolerated. What then can be done? 
In order to answer this question, let us consider first what is the design problem. The problem of design consists of three principal parts which are mechanical design problems and a variable number of other parts which are essentially economic in character. The mechanical design problems are: (1) to define the loads (environmental factors)- which will probably occur on the item being designed, (2) to predict the probable response of the item to these loads, and (3) to judge whether these predicted reeponses are such as to produce the required level of reliability or probability of successful performance of the item's intended function. Some of the economic problems are to fit the item within the limits of size, weight, shape, and cost which are determined by other factors. There are, in fact, many facets of the design problem, and by no means all of these are susceptible to treatment by means of mathematics, testing, and logical reasoning. It would be helpful if at least the noneconomic parts of the problem could be so treated, but to do so would be to ignore the uncertainties involved in the very processes of prediction of loads, responses, or damage and the validity of tests. It is necessary to know a great deal about a design problem before a valid test can be devised and its results interpreted with intelligence.

To be more specific about what can be done, let us first think about the environment that the item will have to live through, from the selection tests for parts and materials, to manufacturing processes, to production testing or quality control, to handling and shipping, to storage, to use, to maintenance or repair. Most of these environmental phases involve a definite, measurable set of conditions or "loads" about which much is 
already known and more can be deduced. These environmental conditions must be translated into numerical values in terms of mean value, extremes, and number and frequency of occurrence.

Once the environmental conditions have been expressed as statistically defined loads, the next step is to study these loads in terms of the kinds of failures or malfunctions they might cause and the relative effect of such a result on the overall reliability. It is reasonable to consider arbitrarily increasing the severity of a load if its effect is to cause a catastrophic failure, if the certainty of correctness (confidence level) of the previously established load value is not high, or if the economic factors involved in providing a high resistance to that load are not limiting. This process amounts to tempering the load values with judgment, just as would be done in interpreting measurements of shock and vibration environments.

The next step is to establish damage criteria. It is necessary to decide in advance and to define in numbers the extent of malfunction or failure that will be considered unacceptable and cause for redesign or rejection just as for the testing process. The means by which these damage criteria will be evaluated need also to be defined, so that alternate designs may be compared on a common basis.

Once the loads have been defined and damage criteria have been established, the design problem reduces to one of predicting or measuring probable responses to loads and evaluating these responses in terms of the established damage criteria. When measurement from tests is not feasible, 
prediction by calculation from data obtained on small models or on similar equipment is the only recourse. It is well to remember, however, that such predictions are not at all the precise processes that they are too often represented to be. Just because desk calculators have many digits on them is no reason to be misled into thinking that a calculation is any better in accuracy as a prediction than the assumptions that went into the calculation.

Now where does this leave us in terms of the question what to do if it is too big to test"? The answer is that all that can be done is to calculate, test small models, use a liberal seasoning of judgment on the results, and hope that the equipment will be kind enough to overlook our errors. With specific regard to shock and vibration problems, basic studies such as were discussed earlier of fer the only hope for reducing the proportion of unknowns for designs which are too big to test. 


\section{References}

1. Murray, W.M. editor: "Fatigue and Fracture of Metals" Technology Press of MIT and Wiley (1952)

2. Proceedings of the International Conference on Fatigue of Metals, Sponsored by Inst. Mech. Eng. \& Am. Soc. Mech. Eng, Sept \& Nov. 1956. (Available at present as separate papers from ASME)

3. Miner, M.A. : Cumulative Damage in Fatigue, Jour. App. Mech. 12(3):159 (1945)

4. Corten, H.T. and T. J. Dolan: Cumulative Fatigue Damage, Session 3, Paper 2, International Conference on Fatigue of Metals, Inst. Mech. Eng. \& Am. Soc. Mech. Eng.

5. "A Guide for Design of Shock Resistant Naval Equipment" NAVSHIPS 250-660-30, Navy Dept., BuShips, 1949.

6. Belsheim, R.0.: Delayed-Yield Time Effect in Mild Steel under Oscillatory Axial Loads, NRL Report 4312 (1954)

7. Mindlin, R.D., F.W. Stubner, and H.L. Cooper: Kesponse of Damped Elastic Systems to Transient Disturbances, Proc. S.E.S.A. V, II, 6, (1948)

8. Goldberg, A. : Study in Extension of Range of Shock-Acceleration of the JAN-S-44 Shock Test Machine, Frankford Arsenal Technical Report, S-3907 (4 Mar 1954)

9. Chapter 4, Laboratory Simulation of Shock and Vibration, "Fundamentals of Guided Missile Packaging", RD 219/3, Department of Defense, July 1955.

10. Johnson, W.F. : Shock Testing Facilities, NOL Report 1056 (Mar 1956)

11. Daniel, R.M. : Simulation of Launching Accelerations on B-62-Missile Cormonents, Northrop Report ETR 7482-1, (24 Ap.1953)

12. Tbid (9) Chapter 4 pp 2I-24.

13. Conrad, R.W. : Characteristics of the Light-Weight High-Impact Shock Machine, NRL Report 3922 (Jan.1952)

14. Conrad, R.W.: Characteristics of Navy Medium-Weight High-Impact Shock Machine, NRL Report 3852 (Sept.1951)

15. Ibid (9) Chapter 5, pp 29-32

16. Unholtz, Ko: The Influence of Blectrical and Motional Impedance on the Control and Performance of Some Vibration Machines, ASME Publication "Shock and Vibration Instrumentation", (June 1956) 


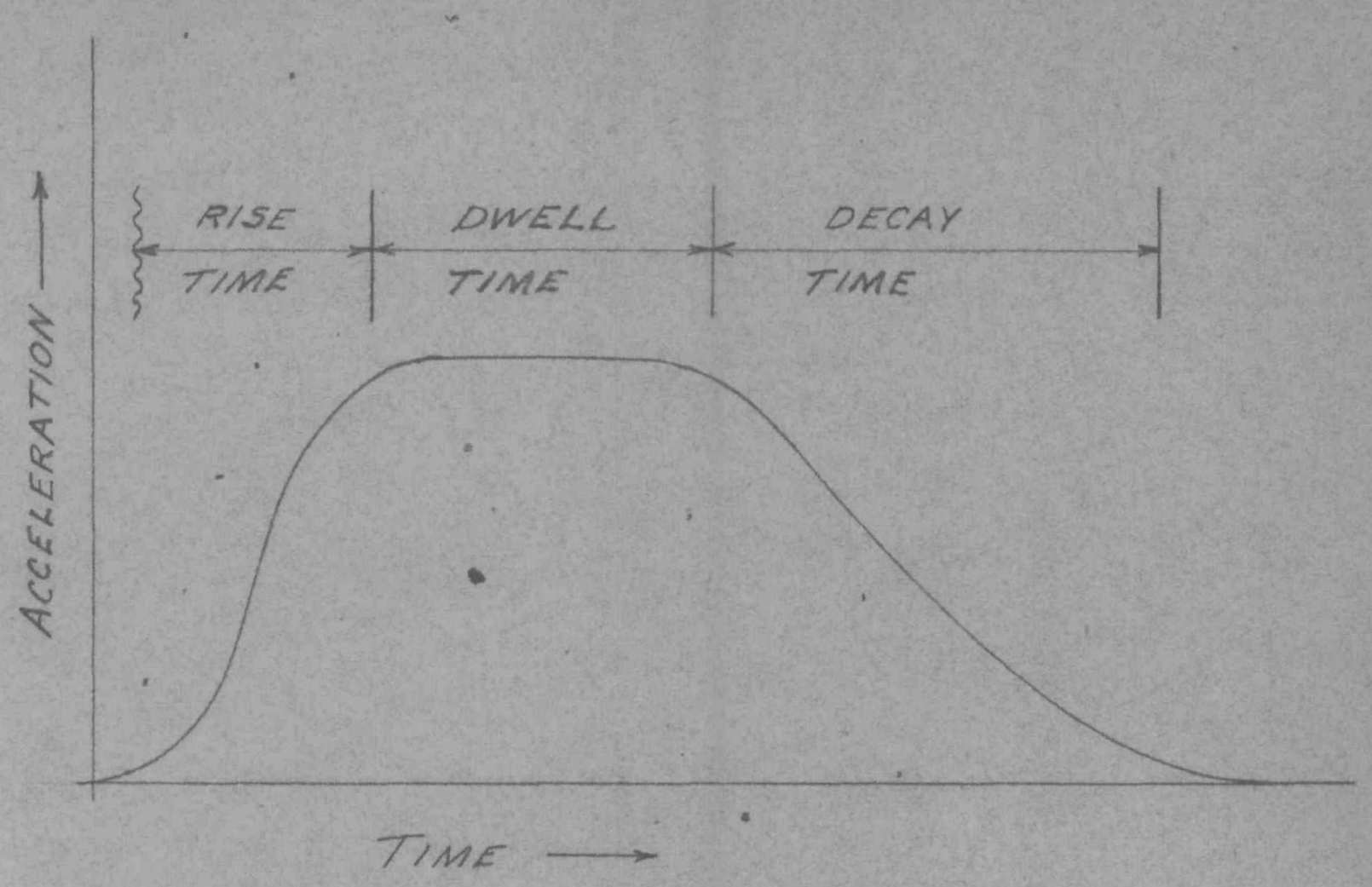

FIG. 24-1. SHOCK-PULSE NOMENCLATURE

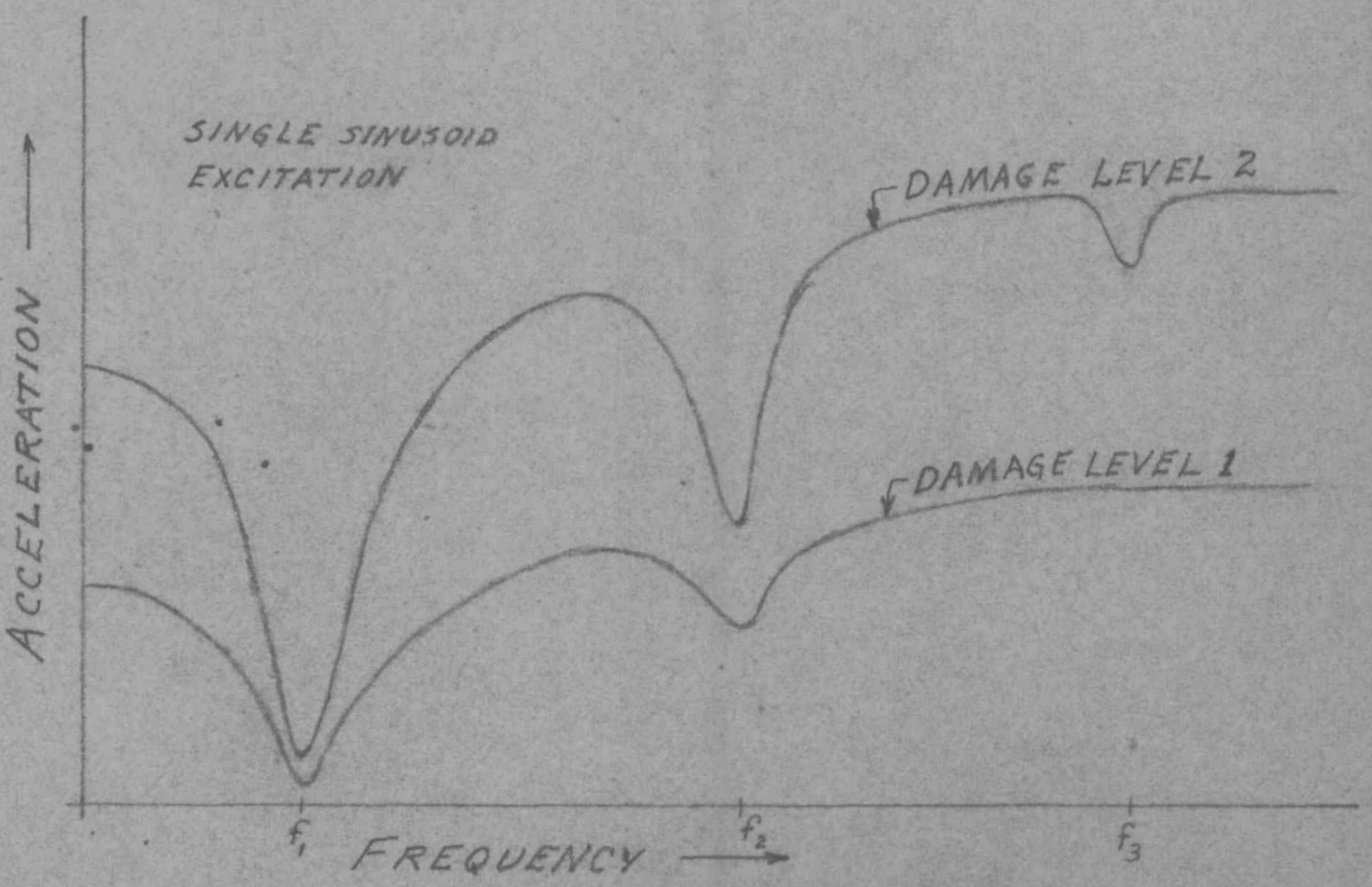

FIG. 24-2. FREQUENCY VS. ACCELERATION FOR! -VARIOUS DAMAGE LEVELS 


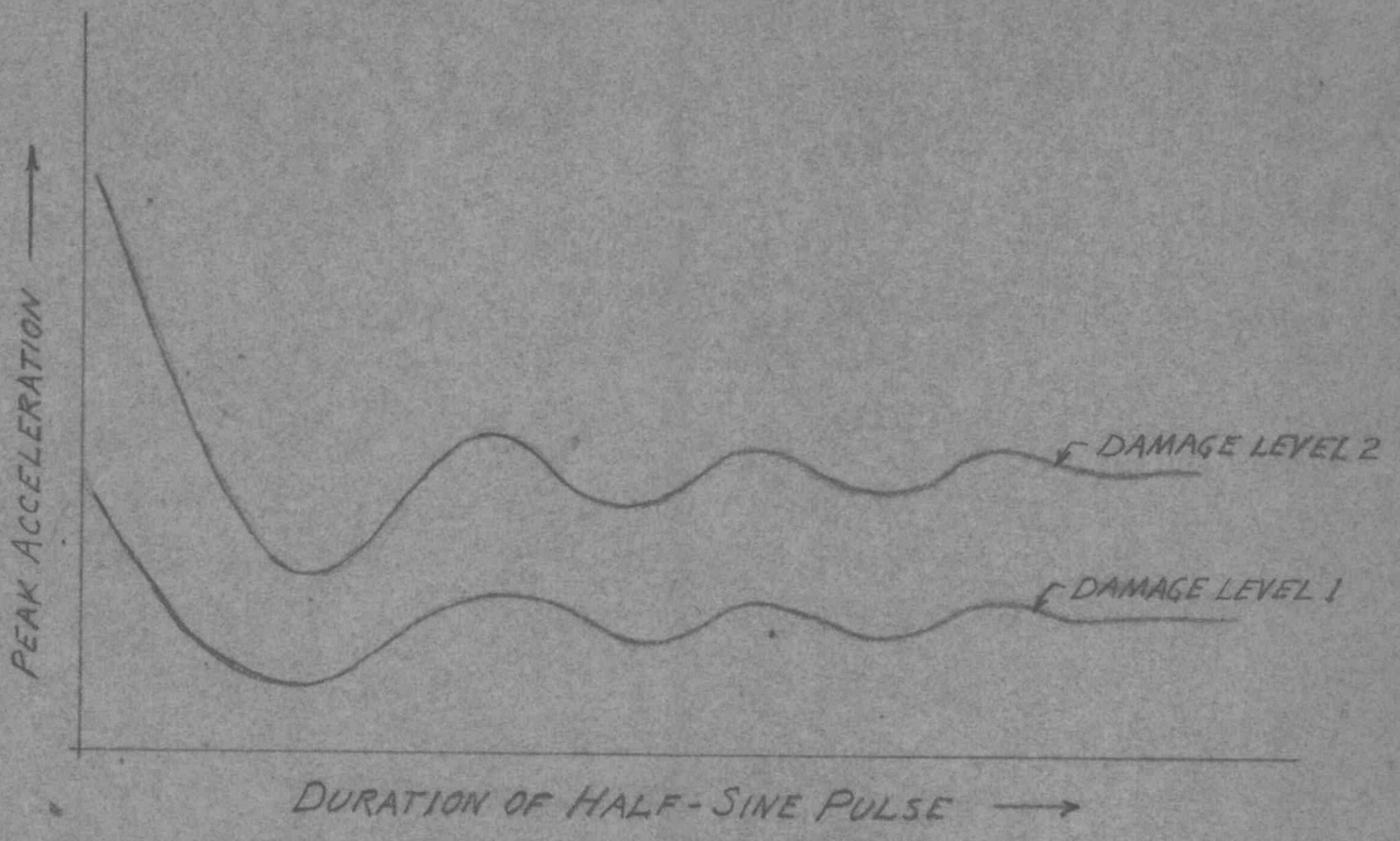

FIG. 24-5. DURATION AND PEAK ACCELERATION OF HALF-SINE PULSE FOR VARIOUS DAMAGE LEVELS 